\title{
ANOMALOUS a.c. RESISTIVITY EFFECT IN Nb AND Sn AT HIGH STATIC FIELDS IN THE LIQUID HELIUM TEMPERATURE RANGE
}

\author{
L. J. M. VAN DE KLUNDERT, A. VAN ARDENNE and L. C. VAN DER MAREL \\ Twente University of Technology, Enschede, The Netherlands
}

\author{
Received 10 March 1971
}

\begin{abstract}
The a.c. susceptibilities of $\mathrm{Nb}$ and $\mathrm{Sn}$ cylinders in the normal state at temperatures below $T_{\mathrm{c}}$ have been measured. The results cannot be described by the formalism of the classical skin-effect. Therefore a formal description is given.
\end{abstract}

In a previous communication [1] we reported susceptibility measurements on a polycrystalline niobium cylinder. It was stated that for fields $H_{0}>H_{\mathrm{c} 3}$ the observed $\chi^{\prime}$ and $\chi^{\prime \prime}$ values did not agree with those predicted by the well-known formula [2]:

$\chi=\chi^{\dagger}+\mathrm{j} \chi^{n}=\frac{2 \mathrm{~J}_{1}(R k)}{R k \mathrm{~J}_{0}(R k)}-1 ;$

$R$ is the radius of the cylinder, $k$ is related to the skin depth $\delta$ by the relation: $k=(1+\mathrm{j}) / \delta$ with

$\delta=(2 / \mu \omega \sigma)^{1 / 2}$.

Since we found that the agreement between relation (1) and the experimental results is perfect e.g. for an impure copper cylinder of the same dimensions with and without an external static field $H_{0}$ the deviation cannot be the result of a demagnetization factor. This agrees with our expectations since the samples are much longer than the pick-up coil.

In this letter we present some new results of experiments on cylindrical samples: niobium single crystal, polycrystalline tin and a tin single crystal (c-axis) parallel to the cylinder axis).

Table 1

Properties of the specimen

\begin{tabular}{l|cccc}
\hline & \multicolumn{4}{|c}{ radius } \\
& $\begin{array}{c}\text { length } \\
(\mathrm{mm})\end{array}$ & $\left.\sigma_{4.2} / \sigma_{300}{ }^{\mathrm{a}}\right)$ & purity $\mathrm{b})$ \\
\hline Nb single crystal & 1.89 & 20.0 & 85 & $6 \mathrm{~N}$ \\
Sn single crystal & 2.90 & 20.1 & $2 \times 10^{4}$ & $6 \mathrm{~N}$ \\
Sn polycrystalline & 2.50 & 20.0 & $7.5 \times 10^{3}$ & $5 \mathrm{~N}$ \\
\hline
\end{tabular}

a) $\sigma$ is the specific conductivity determined from eq. (2) $\sigma_{4.2}$ of $\mathrm{Nb}$ was measured at $H_{0}>7.5 \mathrm{kOe}$.

b) Oxygen content unknown but expected less than $100 \mathrm{ppm}$.
The measurements were performed by an induction method, using a Hartshorn bridge. The sample was placed in a magnetic field $H=H_{0}+$ $h \exp (j \omega t)$ parallel to the cylinder axis. The equipment used in these experiments is the same as already described [1] with one slight modification in order to keep the primary current constant when the external constant field is changed. Previously the magnitude of this current was influenced due to the magnetoresistance of the primary coil. Hence the older measurements should be slightly corrected at high fields but only at the lower frequencies.

A formal description of the results of the measurements can be obtained by putting $R k=$ $r\left(H_{0}, \nu\right) \exp \left\{\mathrm{j} \phi\left(H_{0}, \nu\right)\right\}$ where $r$ and $\phi$ are now functions of the field $H_{0}$ and the frequency related to the complex skin-depth. In this notation $\phi=$ $\frac{1}{4} \pi$ gives the $X^{\prime}$ and $X^{\prime \prime}$ values for the classical skin effect, whereas $\phi=\frac{1}{3} \pi$ represents the anomalous limit. So, for the Sn samples and $H_{0}=0$ the value of $\phi$ is a measure of the anomalous behaviour. For $H_{0}=0$, the $\mathrm{Nb}$ sample is in the superconducting state whereas this is the case for the $\mathrm{Sn}$ specimen at $T<3.7 \mathrm{~K}$. Temperature range for the measurements: 1.6-4.2 K; frequency range: 2-4200 $\mathrm{Hz}$; amplitude a.c. field: 1.86-18.6 Oe; the results are independent of temperature and amplitude.

From relation (1) it can be seen that any set $\left\{x^{\prime}, x^{\prime \prime}\right\}$ values can uniquely represented by a set $\{r, \phi\}$ values. If in eq. (2) $\mu$ and $\sigma$ are real quantities the values of $\phi$ and $r^{2} / \nu$ should be constants with $\phi=\frac{1}{4} \pi$ (classical skin effect) and $r^{2} / \nu=2 \pi R^{2} \mu \sigma$.

However, deviations still are observed which for high fields cannot be ascribed to an anomalous character of the skin effect. Evidently the mean 
free path is much smaller than the skin depth.

The measurements of the niobium sample showed that $X^{\prime}$ and $\chi^{\prime \prime}$ are independent of the field $H_{0}$ when superconductivity is completely destroyed $\left(H_{0}>H_{c 3}\right)$. This indicates that at this temperature $(T=4.2 \mathrm{~K})$ the magnetoresistance is independent of the field and therefore is at a saturation value. The difference between

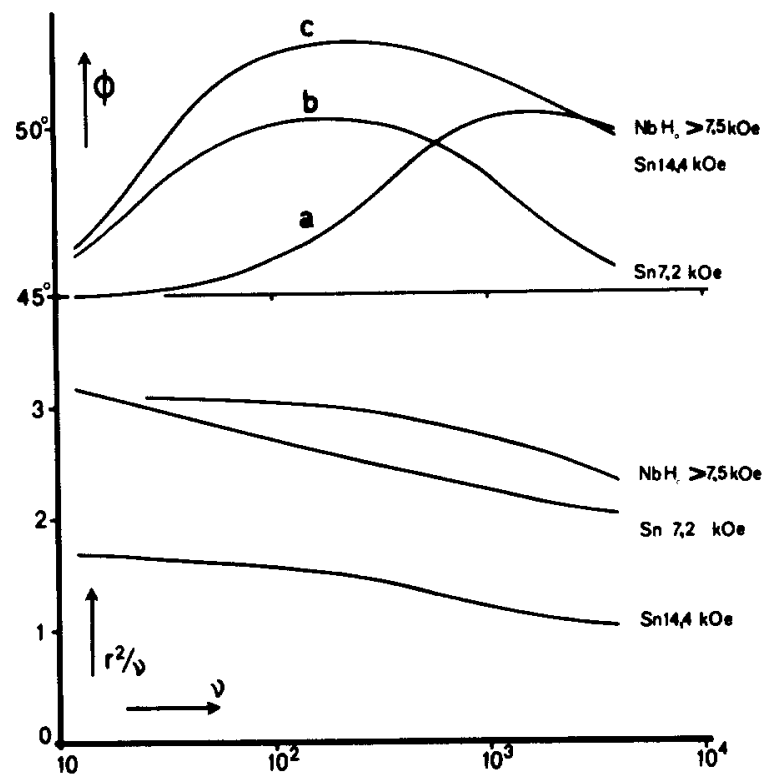

Fig. 1. Frequency dependence of $r^{2} / \nu$ and $\phi$. a) Nb, $\left.T=4.2 \mathrm{~K}, H_{0}>7.5 \mathrm{kOe} . \mathrm{b}\right) \mathrm{Sn}$, polycrystalline, $T=$ $1.6 \mathrm{~K}, H=7.2 \mathrm{kOe}$; c) Sn, polycrystalline, $T=1.6 \mathrm{~K}$, $H=14.4 \mathrm{kOe} . r^{2} / \nu$ in arbitrary units.
$\sigma\left(H_{0}>H_{\mathrm{c} 3}\right)$ and $\sigma\left(H_{0}=0\right)$ obviously cannot be determined.

In fig. 1 the observed values of $r^{2} / \nu$ and $\phi$ have been plotted against $\log \nu$. For the polycrystalline tin sample the behaviour is more complicated. The $X^{\prime}-X^{\prime \prime}$ curves are presented in fig. 2.

Here the $X^{\prime}$ and $X^{\prime \prime}$ values both depend on frequency and field. The corresponding values of $r^{2} / \nu$ have been plotted in fig. 3 against $1 / H_{0}$. The approximate linear behaviour for low $1 / H_{0}$ values indicates that the field dependence of $\sigma$ crudely may be described by $\sigma\left(H_{0}\right)=\sigma(0) /\left(1+a H_{0}\right)$.

In $\mathrm{fig} .4$ the $\phi$ values are given as a function of $H_{0}$. At low field values the excess of $\phi$ is due to the anomalous skin effect, while at high fields possibly a similar mechanism as in the niobium sample is responsible for the anomalous behaviour. This can be seen by plotting, at constant field $H_{0}, r^{2} / \nu$ and $\phi$ against $\log \nu$. Some of these curves are inserted in fig. 1 .

For the tin single crystal the difference $\phi-$ $\frac{1}{4} \pi$ is at least one order smaller than in the order specimen at high fields. The behaviour is masked by the De Haas-Shubnikov oscillations; the two lower frequencies of the oscillations could be determined and are in agreement with values given by Stafleu and De Vroomen [3].

Since in the tin single crystal at high fields no deviations are observed $\left(\phi=\frac{1}{4} \pi\right)$, it is suggested that the orientation of the external field to the Fermi surface (anisotropic both for $\mathrm{Nb}$ and $\mathrm{Sn}$ ) plays a role. The experiments will be continued with other single crystals of $\mathrm{Sn}$ with different orientation of the $c$-axis and with $\mathrm{Nb}$ and $\mathrm{Cu}$ crystals. The results will be reported.

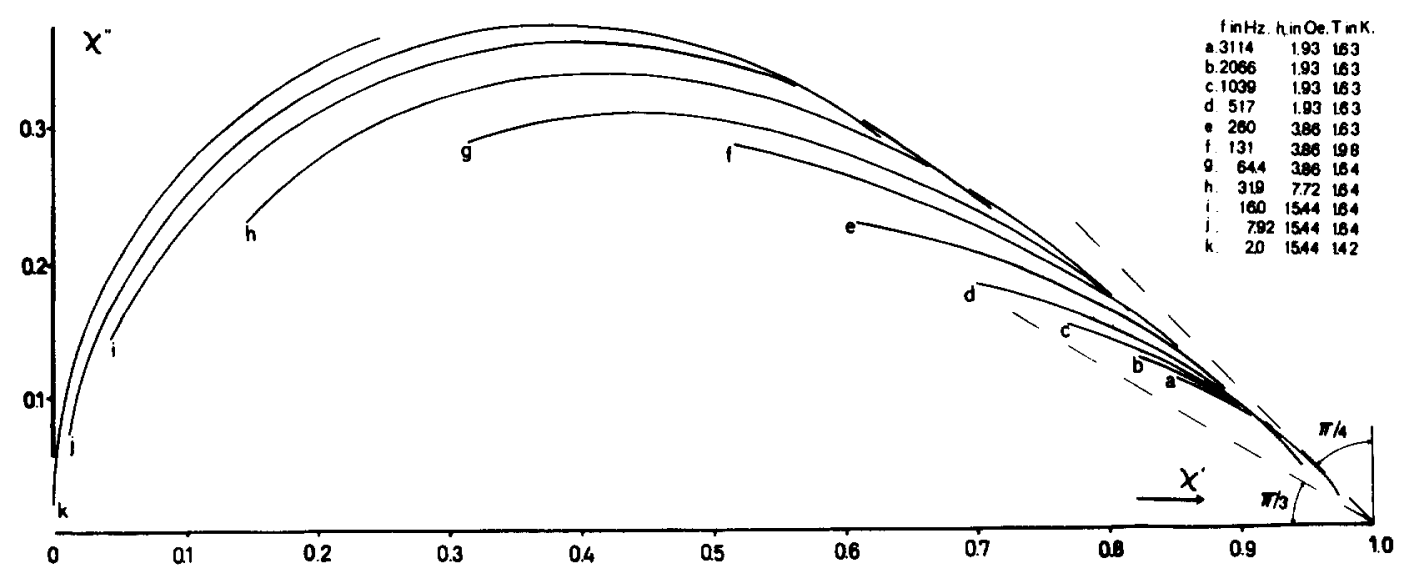

Fig. 2. The $X^{\prime}$ versus $X^{n}$ curves of polycrystalline $\mathrm{Sn}$ at different frequencies. The parameter along each curve is the external field $H_{0}$ amounting to $20 \mathrm{kOe}$. Increasing field corresponds to lower $\left|X^{\prime}\right|$ values. 


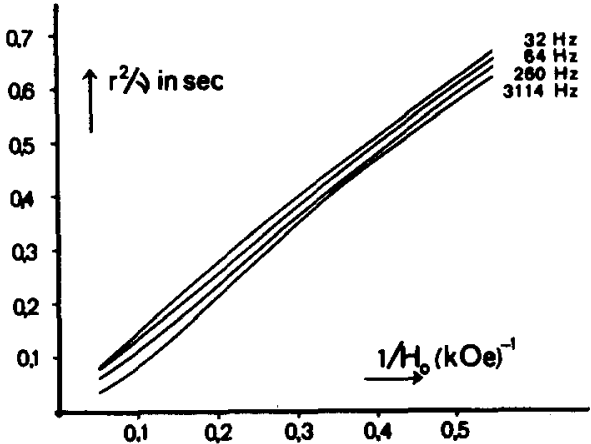

Fig. 3. $r^{2} / \nu$ values as a function of $1 / H_{0}$ for polycrystalline Sn.

The single crystals were obtained from the "Hollandse Metallurgische Industrie Billiton", Arnhem, The Netherlands and the polycrystalline material from Johnson Matthey Chem. Ltd., England.

We gratefully acknowledge the technical assistance of Mr. J. A. Ulfman, and are indebted

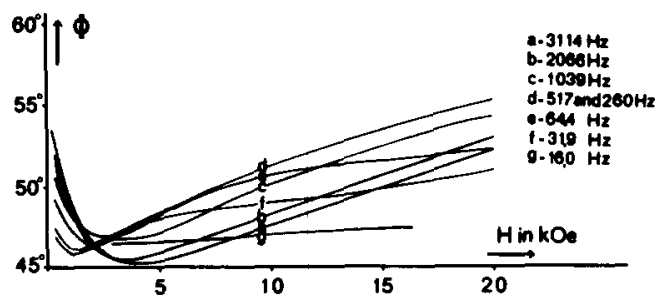

Fig. 4. $\phi$ as a function of $H_{0}$ and frequency for polycrystalline $\mathrm{Sn}$.

to Mr. J.A. de Jong for performing some of the calculations.

\section{References}

[1] L. J. M. van de Klundert, G. E. Alberga, L. C.van der Marel and C. Leijnse, Proc. third Intern. Cryogenic engineering Conf., Berlin 1970, p. 346.

[2] L. D. Landau and E. M. Lifshitz, Electrodynamics of continuous media, Vol. 8 (Pergamon Press, Oxford, 1960) p. 194.

[3] M.D.Stafleu and A.R. de Vroomen, Phys. Letters 23 (1966) 179.

\section{AN INEQUALITY FOR THE THERMAL HEAT CAPACITY AT CONSTANT MAGNETIZATION IN THE ISING MODEL}

V. K. FEDJANIN

The Karpov Institute of Physical Chemistry, Moscow, USSR

Received 12 March 1971

A new inequality for $C_{M}$ has been obtained with the help of the inequality for the first and second correlation functions in the Ising model, $f_{1}$ and $f_{2}$.

As has been shown in ref. [1] for $f_{1}$ and $f_{2}$ the following strong inequality holds

$$
\begin{aligned}
& L+2 z I f_{2} \geqslant(2 L+z I) f_{1}+2 \sigma^{2} / \beta, \\
& \beta=(k T)^{-1}, \quad L=\mu H+\frac{1}{2} z I, \\
& \sigma=1-2 f_{1}
\end{aligned}
$$

where $H$ is the magnetic field, $I$ is the exchange interaction. Inequality (1) holds good in the whole range of temperatures $\beta$ and magnetic fields $H$ : $0<\beta<\infty, 0 \leqslant H<\infty$.

Dismissing the last term and rearranging the terms remained we have

$F \geqslant G, \quad F=2 z l f_{2}, \quad G=z I f_{1}-L \sigma$.

Let us consider the derivatives $F$ and $G$ by $\beta$ at fixed magnetization $M$ of the system $\left(M=\frac{1}{2} \mu N \sigma\right)$, that is at fixed $f_{1}(\beta, H)$. In this case the magnetic field $H$ is a function of $\beta$ and 